\title{
SOLVABLE AND NILPOTENT NEAR-RING MODULES ${ }^{1}$
}

\author{
GORDON MASON
}

\begin{abstract}
The center of a unital near-ring module ${ }_{n} M$ is defined, leading to the construction of a lower central series and a definition of $R$-nilpotence. Likewise a suitable definition of commutators yields a derived series and $R$-solvability. When $(R,+)$ is generated by elements which distribute over $M$ the $R$-nilpotence ( $R$-solvability) is shown to coincide with the nilpotence (solvability) of the underlying group. In this case, nilpotence has implications for $R$-normalizers and the Frattini submodule.
\end{abstract}

1. Introduction. For basic definitions see [2] or [3]. In this paper, by "near-ring" is meant a right unital near-ring $R$ satisfying $x \cdot 0=0$ for all $x \in R$. Similarly a (left) near-ring module ${ }_{R} M$ over $R$ will always be assumed to be unital. In general a subgroup $A$ of $(M,+)$ is called an $R$-subgroup if $R A \subseteq A$, and $A$ is an $R$-submodule if it is a normal $R$-subgroup satisfying

$$
\text { For all } r \in R, m \in M, a \in A, \quad r(m+a)-r m \in A .
$$

In the unital case, a subgroup with property (SM) is in fact a normal subgroup.

ISOMORPHISM THEOREM [2]. Let $f: M \rightarrow M^{\prime}$ be an R-epimorphism.

(i) If $A$ is an $R$-subgroup ( $R$-submodule) of $M$, then $f(A)$ is an $R$ subgroup ( $R$-submodule) of $M^{\prime}$.

(ii) If $A^{\prime}$ is an $R$-subgroup ( $R$-submodule) of $M^{\prime}$ then $f^{-1}(A)$ is an $R$-subgroup ( $R$-submodule) of $M$.

(iii) If $A$ is an $R$-subgroup ( $R$-submodule) of $M$ containing $\operatorname{ker} f$, $f^{-1}(f(A))=A$.

A normal series for $M$ is a finite series $M \supset M_{1} \supset \cdots \supset M_{n}=0$ where each $M_{i}$ is an R-submodule of $M_{i-1}$. Any two normal series for $M$ have equivalent refinements. $M$ is called simple if it has no proper $R$-submodules and irreducible if it has no proper $R$-subgroups. A composition series is a normal series without repetition whose factors are all simple. The Jordan-Hölder theorem holds.

Received by the editors July 25,1972 .

AMS (MOS) subject classifications (1970). Primary 16A76.

Key words and phrases. Near-ring module, center, solvable, nilpotent.

${ }^{1}$ This research was supported in part by the National Research Council of Canada. 
2. Nilpotence. Define the center of ${ }_{R} M$ to be $Z(M)=Z_{R}(M)=$ $\{a \in M \mid r(b+s a)=r s a+r b$ for all $b \in M, r, s \in R\}$. Taking $r=s=1$ we see $Z_{R}(M) \subseteq Z_{Z}(M)$. For $x, y \in M$ and $r, s \in R$ define $[y, x, r, s]=$ $r(y+s x)-r y-r s x$ and define the upper central series inductively by $Z_{0}=0, Z_{1}=Z(M), Z_{i}=\left\{x \in M \mid[y, x, r, s] \in Z_{i-1}\right.$ for all $\left.y \in M, r, s \in R\right\}$. Clearly these sets satisfy $Z_{i} \subseteq Z_{i+1}$ for all $i$.

THEOREM 2.1. $Z_{i}$ is an $R$-submodule of $M$ for all $i$ and $Z_{i} / Z_{i-1}=$ $Z\left(M / Z_{i-1}\right)$.

Proof. Inductively, assume $Z_{i-1}$ is an $R$-submodule. We first show that $Z_{i}$ is a subgroup of $M$. If $x, x^{\prime} \in Z_{i}$ then there exist $z_{j} \in Z_{i-1}$ for $j=1,2, \cdots, 7$ such that

$$
\begin{aligned}
r\left(y+s\left(x-x^{\prime}\right)\right) & =r\left(y+z_{1}+s(-1) x^{\prime}+s x\right) \\
& =z_{2}+r s x+r\left(y+z_{1}+s(-1) x^{\prime}\right) \\
& =z_{2}+r s x+z_{3}+r s(-1) x^{\prime}+r\left(y+z_{1}\right) \\
& =z_{2}+r s x+z_{3}+r s(-1) x^{\prime}+z_{4}+r y \\
& =z_{5}+r s x+r s(-1) x^{\prime}+r y \\
& =z_{5}+\left[z_{6}+r s(-1) x^{\prime}+r s x\right]+r y \\
& =z_{5}+z_{6}+z_{7}+r s\left(x-x^{\prime}\right)+r y .
\end{aligned}
$$

Hence $r\left(y+s\left(x-x^{\prime}\right)\right)-r y-r s\left(x-x^{\prime}\right) \in Z_{i-1}$ as required. Moreover $Z_{i}$ is clearly an $R$-subgroup. Also $Z_{1}$ has property (SM) since, if $a \in Z(M)$, $r \in R$ and $b \in M$, then $r(b+a)-r b=r a+r b-r b=r a \in Z(M)$. Now suppose inductively that $Z_{i-1} / Z_{i-2}=Z\left(M / Z_{i-2}\right)$. Then $x \in Z_{i}$ iff $[y, x, r, s] \in Z_{i-1}$ iff $\left[y+Z_{i-1}, x+Z_{i-1}, r, s\right]=Z_{i-1}$ for all $y+Z_{i-1} \in M / Z_{i-1}$. Hence $Z_{i} / Z_{i-1}=Z\left(M / Z_{i-1}\right)$ as claimed, and this shows also that $Z_{i} / Z_{i-1}$ is an $R$-submodule of $M / Z_{i-1}$. By the isomorphism theorem therefore, $Z_{i}$ is an $R$-submodule of $M$.

Remark. An easy calculation shows that $Z(M)$ is the categorical center as defined in [1].

Define $M$ to be $R$-nilpotent of class $n$ if $n$ is the least integer such that $Z_{n}=M$.

Writing $[y, x, r, 1]=[y, x, r]$ we define the $R$-commutator of two $R$-subgroups $A$ and $B$ to be the $R$-subgroup $[A, B]_{R}$ (or $[A, B]$ ) generated by $\{[a, b, r] \mid a \in A, b \in B, r \in R\}$.

Proposition 2.2. If $B$ is an $R$-submodule of $A \subseteq M$, then $[A, B]$ is an $R$-submodule of $A$.

Proof. By definition, $[A, B]$ is an $R$-subgroup of $M$. Since $B$ is an $R$-submodule of $A, r(a+b)-r a \in B$ so $[a, b, r] \in B \subset A$ and $[A, B]$ is 
an $R$-subgroup of $A$. Finally

$$
\begin{aligned}
r\left(a+\left[a_{1}, b, s\right]\right)-r a & =r\left(a+b_{1}\right)-r a, \quad \text { where }\left[a_{1}, b, s\right]=b_{1} \in B \\
& =r\left(a+b_{1}\right)-r a-r b_{1}+r b_{1} \\
& =\left[a, b_{1}, r\right]+r\left[a_{1}, b, s\right] \in[A, B]
\end{aligned}
$$

for all $a \in A, r \in R$ so the condition (SM) holds.

Define $M_{R}^{\prime}=[M, M]_{R}$. By the proposition, $M^{\prime}$ is an $R$-submodule of $M$, and in fact $R^{\prime}$ is an ideal of $R$. Define $M$ to be central if $M=Z_{R}(M)$ (iff $M^{\prime}=0$ ).

THEOREM 2.3. (a) $M / M^{\prime}$ is central and if $A$ is an $R$-submodule of $M$, $M / A$ is central iff $A \supseteq M^{\prime}$.

(b) If $N$ is an $R$-subgroup of $M$ and $N \supseteq M^{\prime}$, then $N$ is an $R$-submodule.

Proof. (a) $A \supseteq M^{\prime}$ iff $[x, y, r] \in A$ for all $r \in R, \quad x, y \in M$ iff $[x+A, y+A, r]=0$ in $M / A$ for all $r \in R, x, y \in M$ iff $[M / A, M / A]=0$.

(b) $N / M^{\prime}$ is an $R$-subgroup of $M / M^{\prime}$ by the isomorphism theorem and $M / M^{\prime}$ is central. Therefore $N / M^{\prime}$ is an $R$-submodule of $M / M^{\prime}$ and so $N$ is an $R$-submodule of $M$.

REMARK 1. Since $M$ is unital we see $M$ is central iff it is abelian and $R$ is distributive over $M$.

RemarK 2. In general $[M, M]_{Z} \neq[M, M]_{R}$. For example if $G$ is an abelian group, $G$ is a module over the near-ring $R=$ maps $f: G \rightarrow G \mid$ $f(0)=0\}$. Clearly $[G, G]_{Z}=0$, but $[G, G]_{R}=D(G)$ the distributor submodule which is nonzero as $R$ is not distributive over $G$.

Define as usual Ann $M=\{r \in R \mid r M=0\}$. Then Ann $M$ is an ideal in $R$. If Ann $M=0$, call $M$ faithful.

Proposition 2.4. If there exists a faithful central $R$-module $M$, then $R$ is a ring.

Proof. Since $(r+s) m=r m+s m=s m+r m=(s+r) m$ for all $m \in M$ and similarly $r(s+t)-(r s+r t) \in$ Ann $M=0$, therefore $R$ is abelian and distributive and so is a ring.

Define the lower central series inductively by $Z^{(0)}=M, Z^{(i)}=$ $\left[M, Z^{(i-1)}\right]_{R}$. By Proposition 2.2 each $Z^{(i)}$ is an $R$-submodule of $M$.

THEOREM 2.5. $\quad M$ is R-nilpotent of class $n$ iff $n$ is the least integer such that $Z^{(n)}=0$.

Proof. We first prove $Z^{(i)} \subseteq Z_{n-i}$. Inductively, since $Z^{(0)}=Z_{n}=M$, suppose $Z^{(i-1)} \subseteq Z_{n-i+1}$. Then $Z_{n-i} \supseteq\left[M, Z_{n-i+1}\right] \supseteq\left[M, Z^{(i-1)}\right]=Z^{(i)}$. Hence 
$Z^{(n)} \subseteq Z_{0}=0$. Moreover $n$ is minimal for if $Z^{(n-1)}=0$ then using the series $Z^{(n-1)} \subset \cdots \subset Z^{(0)}$ we can show as above that $Z^{(i)} \subseteq Z_{n-1-i}$. Therefore $M=Z^{(0)} \subseteq Z_{n-1}$ which contradicts the minimality of $n$ in the upper central series. The converse is proved similarly.

3. Solvability. Define the derived series for $M$ inductively by $M^{(1)}=$ $[M, M]_{R}, M^{(i)}=\left[M^{(i-1)}, M^{(i-1)}\right]_{R}$, and define $M$ to be $R$-solvable if $M^{(n)}=0$ for some $n$. Since inductively $M^{(i)} \subset Z^{(i)} \Rightarrow M^{(i+1)}=\left[M^{(i)}, M^{(i)}\right] \subseteq$ $\left[M, Z^{(i)}\right]=Z^{(i+1)}$ it follows that if $M$ is $R$-nilpotent, it is $R$-solvable.

It is clear from the definitions that if $M$ is $R$-nilpotent ( $R$-solvable) it is nilpotent (solvable) as a group. In fact if $f: S \rightarrow R$ is a near-ring homomorphism and ${ }_{R} M$ is canonically an $S$-module then $R$-nilpotence $(R$ solvability) implies $S$-nilpotence ( $S$-solvability).

THEOREM 3.1. $M$ is $R$-solvable iff $M$ has a normal series whose factors are all central.

Proof. If $M$ is $R$-solvable then $M^{(n)}=0$, so the series $\left\{M^{(i)}\right\}$ is a normal series in view of Proposition 2.2. Moreover each factor is central by Theorem 2.3. Conversely, suppose $M \supset M_{1} \supset \cdots \supset M_{n}=0$ is a normal series for which $M_{i} / M_{i+1}$ is central for all $i$. By induction if $M^{(i)} \subset M_{i}$, then $M^{(i+1)}=\left[M^{(i)}, M^{(i)}\right] \subset\left[M_{i}, M_{i}\right] \subset M_{i+1}$ by Theorem 2.3. Therefore $M_{n}=0 \Rightarrow M^{(n)}=0$.

Proposition 3.2. (a) Every $R$-subgroup $A$ and every factor module $M / B$ of an $R$-solvable module $M$ is $R$-solvable.

(b) If $B$ is an $R$-solvable $R$-submodule of $M$ and $M / B$ is $R$-solvable then $M$ is $R$-solvable.

Proof. (a) The canonical monomorphism $\alpha: A \rightarrow M$ and epimorphism $\beta: M \rightarrow M / B$ induce respectively monomorphisms $\alpha^{(k)}: A^{(k)} \rightarrow M^{(k)}$ and epimorphisms $\beta^{(k)}: M^{(k)} \rightarrow(M / B)^{(k)}$.

(b) Given $0 \rightarrow B \rightarrow^{\alpha} M \rightarrow^{\pi} M / B \rightarrow 0,(M / B)^{(k)}=0$ implies $\pi$ restricts to $\pi^{(k)}: M^{(k)} \rightarrow 0$ so $M^{(k)} \subset \operatorname{ker} \pi=\operatorname{Im} \alpha$. Since $B^{(m)}=0,\left(M^{(k)}\right)^{(m)}=M^{(k+m)}=0$.

4. The distributively generated case. In this section we shall assume that $R$ is distributively generated (d.g.) over $M$, by which we mean that there exists a multiplicative semigroup $S \subseteq R$ such that $s(m+n)=s m+s n$ for all $s \in S, m, n \in M$ and such that $S$ additively generates $R$. In this case we note that $s(-m)=-s m$ for all $s \in S, m \in M$ and also a normal $R$ subgroup of $M$ is an $R$-submodule. Clearly $Z_{R}(M) \subseteq\{a \mid b+s a=s a+b$ for all $s \in R, b \in M\}$ and, when $R$ is d.g. over $M$, equality holds. For suppose $r \in R$ and $r=t_{1}+t_{2}$ where the $t_{i}$ are distributive over $M$ (the proof goes inductively for $r=\sum_{i=1}^{n} t_{i}$ ). Then if $b+s a=s a+b$ for all $b \in M$, 
$s \in R$ we have

$$
\begin{aligned}
r(b+s a) & =t_{1}(b+s a)+t_{2}(b+s a)=t_{1} b+t_{1} s a+t_{2} b+t_{2} s a \\
& =t_{1} b+t_{2} b+t_{1} s a+t_{2} s a=r b+r s a=r s a+r b,
\end{aligned}
$$

so $a \in Z(M)$.

Thus $Z_{R}(M)=M$ iff $Z_{Z}(M)=M$ so that $M$ is $R$-nilpotent of class 1 iff $M$ is nilpotent of class 1 . In fact we shall show that $R$-nilpotence $(R$ solvability) is equivalent to $Z$-nilpotence ( $Z$-solvability). The main results depend on the following group-theoretic lemma.

Lemma 4.1. Let $G$ be a group (written multiplicatively) and let $A, B$ be normal subgroups. Then for every integer $n$, for all $a_{i} \in A, b_{i} \in B$

$$
\left(\prod_{1}^{n} b_{i} a_{i}\right)\left(\prod_{1}^{n} b_{i}\right)^{-1}\left(\prod_{1}^{n} a_{i}\right)^{-1} \in[A, B]
$$

Proof. First note that for all $g \in G, a \in A, b \in B \quad g[a, b] g^{-1}=$ $\left[\mathrm{gag}^{-1}, \mathrm{gbg}^{-1}\right] \in[A, B]$. Then for all $a_{1} \in A, b_{1} \in B$

$$
a_{1} b_{1}[a, b] a_{1}^{-1} b_{1}^{-1}=\left[a_{1}, b_{1}\right] b_{1} a_{1}[a, b]\left(b_{1} a_{1}\right)^{-1} \in[A, B] .
$$

For $n=2$,

$$
b_{1} a_{1} b_{2} a_{2} b_{2}^{-1} b_{1}^{-1} a_{2}^{-1} a_{1}^{-1}=b_{1} a_{1}\left[b_{2}, a_{2}\right]\left[a_{2}, b_{1}^{-1}\right] b_{1}^{-1} a_{1}^{-1} \in[A, B] .
$$

For $n \geqq 3$ the result comes from repeated application of $\left({ }^{*}\right)$ and the identity $\left(\prod_{1}^{n} b_{i}\right)^{-1}\left(\prod_{1}^{n} a_{i}\right)^{-1}=\left(\prod_{i=n}^{3} b_{i}^{-1} a_{i}^{-1}\left[a_{i}, \prod_{i-1}^{2} b_{j}^{-1}\right]\right) b_{2}^{-1} a_{2}^{-1}\left[\prod_{2}^{n} a_{i}, b_{1}^{-1}\right] b_{1}^{-1} a_{1}^{-1}$.

Theorem 4.2. $[A, B]_{R}=[A, B]_{Z}$ if $A$ and $B$ are $R$-submodules of $M$.

Proof. Since $1 \in R$, every generator of $[A, B]_{Z}$ is in $[A, B]_{R}$. Conversely if $y=r(a+b)-r a-r b$ is a generator of $[A, B]_{R}$ and $r=\sum_{1}^{n} s_{i}$, $s_{i} \in S$ then

$$
y=\sum_{i=1}^{n}\left(s_{i} a+s_{i} b\right)-\left(\sum_{1}^{n} s_{i} a\right)-\left(\sum_{1}^{n} s_{i} b\right) \in[A, B]_{Z}
$$

by the (additive form of the) lemma. Since $[A, B]_{Z}$ is an $R$-subgroup in the d.g. case, the result follows.

Corollary 1. If $A$ and $B$ are $R$-submodules of $M,[A, B]_{R}=[B, A]_{R}$ and this is an R-submodule of $M$.

Corollary 2. $M$ is R-nilpotent (R-solvable) iff $M$ is Z-nilpotent (Z-solvable).

Corollary 3 [3, Theorem 4.4.3]. $R$ abelian and d.g. $\Rightarrow R$ is a ring. 
$R$-solvability can be expressed in terms of an $R$-composition series as follows. Following [4] we define an ideal $P$ of $R$ to be prime if whenever $A, B$ are ideals such that $A B \subseteq P$ then $A \subseteq P$ or $B \subseteq P$ (here $A B$ refers to the additive group generated by all $a b$ ).

Proposition 4.3 [4]. If $M$ is a cyclic simple module, Ann $M$ is a prime ideal.

Proposition 4.4. If $M$ is $R$-solvable and has a composition series, the series has cyclic factors $A_{i}$ where Ann $A_{i}$ is a prime ideal.

Proof. $\quad M$ has a normal series with central factors. By Theorem 2.3 this can be refined to a composition series with central simple factors i.e. central irreducible factors which are therefore cyclic and so have prime annihilators by 4.3 .

We shall now investigate some consequences of $R$-nilpotence.

Let $A$ be an $R$-subgroup of $M$. Define the $R$-normalizer of $A$ to be $N_{R}(A)=N(A)=\{x \in M \mid r x+a-r x \in A$ for all $a \in A, r \in R\}$.

Proposition 4.5. (a) $N(A)$ is an R-subgroup of $M$. (b) $N(A)$ is the largest $R$-subgroup of $M$ in which $A$ is an $R$-submodule.

Proof. (a) Consider, for $x, y \in N(A), z=r(x-y)+a-r(x-y)$ where $r=\sum_{1}^{n} s_{i}, s_{i} \in S$. By induction on $n$,

$$
\begin{aligned}
z & =s_{1} x-s_{1} y+\left(\sum_{2}^{n} s_{i}\right)(x-y)+a-\left(\sum_{2}^{n} s_{i}\right)(x-y)+s_{1} y-s_{1} x \\
& =s_{1} x-s_{1} y+a^{\prime}+s_{1} y-s_{1} x, \quad a^{\prime} \in A,
\end{aligned}
$$

so $z \in A$. Clearly $x \in N(A) \Rightarrow t x \in N(A)$ for all $t \in R$.

(b) $A$ is clearly a normal subgroup of $N(A)$ so it is an $R$-submodule since $M$ is d.g. If $A$ is an $R$-submodule of $B \subset M$ then, for all $b \in B$, $r \in R, r b \in B$; so by the normality of $A$ in $B, r b+a-r b \in B$ for all $a \in A$ and hence $b \in N(A)$, i.e. $B \subseteq N(A)$.

Proposition 4.6. If $M$ is nilpotent and $A$ is an $R$-subgroup of $M$ then $A \subsetneq N(A)$.

Proof. If $k$ is the largest integer such that $Z_{k} \subset A$, choose $x \in Z_{k+1}$, $x \notin A$. Then for all $a \in A, r \in R, a+[-a, x, 1, r]=r x+a-r x \in A+Z_{k} \subset A$ so $x \in N(A)$.

COROLlary. If $M$ is nilpotent, every maximal $R$-subgroup of $M$ is an R-submodule.

Lemma 4.7. A cyclic $R$-module $R m$ is central iff $R^{\prime} \subset$ Ann $m$. 
Proof. $R m$ is central iff $x+s y=s y+x$ for all $x, y \in R m, s \in R$ iff $(r+s t) m=(s t+r) m$ for all $r, s, t \in R$ iff $r+s t-(s t+r) \in$ Ann $m$ iff $[R, R] \subset$ Ann $m$.

Define the Frattini subgroup of $M$ to be

$$
\begin{aligned}
F(M) & =F_{R}(M)=\bigcap\{\text { maximal proper } R \text {-subgroups of } M\} \text { if any exist } \\
& =M \text { otherwise. }
\end{aligned}
$$

Thus by universal algebra, $F(M)$ is the set of nongenerators of $M$.

Proposition 4.8. If $F(M) \supset M^{\prime}$, every maximal $R$-subgroup of $M$ is an $R$-submodule and when $R^{\prime} \subset$ Ann $M$ the converse is true.

Proof. $F(M) \supset M^{\prime}$ implies $A \supset M^{\prime}$ for every maximal $R$-subgroup $A$. By Theorem 2.3, $A$ is an $R$-submodule. Conversely if every maximal $R$-subgroup $A$ is an $R$-submodule then $M / A$ is an irreducible $R$-module so is cyclic. Writing $M / A=R a, R^{\prime} \subset$ Ann $M \subset$ Ann $(M / A) \subset$ Ann $a$ implies by the lemma that $M / A$ is central. Thus $A \supset M^{\prime}$ and as this is true for all maximal $R$-subgroups $A, F(M) \supset M^{\prime}$ as required.

THEOREM 4.9. If $M$ is nilpotent and $R^{\prime} \subset$ Ann $M$ then $M^{\prime} \subset F(M)$.

Proof. If $M$ is nilpotent, every maximal $R$-subgroup is an $R$ submodule by the corollary to 4.6 so $M^{\prime} \subset F(M)$ by Proposition 4.8 .

\section{REFERENCES}

1. M. Barr, What is the center? Reports of the Midwest Category Seminar III, Lecture Notes in Math., vol. 106, Springer-Verlag, Berlin and New York, 1969, pp. 1-12.

2. J. Beidleman, On near-rings and near-ring modules, Doctoral Thesis, Pennsylvania State University, University Park, Pa., 1964.

3. A. Frohlich, Distributively generated near-rings, I. Ideal theory, Proc. London Math. Soc. (3) 8 (1958), 76-94. MR 19, 1156.

4. R. Laxton, Prime ideals and the ideal-radical of a distributively generated near-ring, Math. Z. 83 (1964), 8-17. MR 28 \#3057.

Department of Mathematics, The University of New Brunswick, Fredericton, New BRunswick, Canada 